\title{
The Edge Detection of Steel Cord Pieces Based on Line Fitting
}

\author{
Qinzhou Niu', Lu Yuan² \\ College of Information Science and Engineering, Guilin University of Technology, Guilin 541006, \\ China \\ E-mail:503884266@qq.com
}

Keywords: Line fitting; Edge detection; Image processing; Steel cord.

Abstract: According to the high speed visual inspection requirements of steel cord pieces cut by steel cord body ply cutter( produced by a processing plant) about edge size and angle, this paper proposed a new method of edge detection based on line fitting method. The method solved the error problem of steel cord pieces' rough selvedge, effectively suppress the influence of noise, avoided the disadvantage of the traditional algorithm which has large computation, fully considered the gray information of the interior point in the edge transition region and realized the accurate fitting. Experimental results and analysis show that the method can ensure the detection precision, and greatly improve detection effectiveness.

\section{Introduction}

Steel cord is mainly used in skeleton material of tire and adhesive tape products, is the basis of tire production. Steel cord, after cut by the steel cord body ply cutter with a certain angle and width , require for docking and stitch joint which need be stitched evenly. The size of the cutting piece must meet the requirements; it will cause the following results if there is a large deviation: the tire products unqualified or degradation. [1,2] The edge detection of steel cord is to detect length and angle of stitching edge and to ensure that the size of steel cord pieces meet the requirements in order to ensure the accurate suture of two pieces of steel cord.

The steel cord pieces picture is acquired in real time by CCD camera, accuracy and speed of image processing, to a great extent, determines the accuracy and effect of the system. In industrial production, the product, usually need to achieve high precision of edge detection and morphological detection, the detection accuracy is generally accurate to $0.1 \mathrm{~mm}$ or less. For the types of industrial cutting pieces, the image operation we need first is the image edge extraction of cutting pieces, and then calculate the size and angle according to the processing results of the edge.

\section{The traditional edge detection method}

For digital image, we usually use the convolution operation to deal with the gray pixels in the field or operations like convolution to analyze the gray level difference, to identify the edges of image regions, the common methods are: Roberts gradient, Sobel operator, Laplacian, Canny operator and so on.

When using edge operator to detect the edge of the digital image region, the edge is defined as a set of gray pixels:

$$
\{x \mid F(x)>J\}
$$

$\mathrm{X}$ is the independent pixel number, $\mathrm{F}(\mathrm{x})$ is the calculation results of the operator, $\mathrm{J}$ is the critical value of gray edge. From the definition we can see that the edge detection process needs to calculate each pixel of the image respectively, so as to determine whether it is the edge set.[3]

As the standard method of general edge extraction, operator method has obvious advantages, that is, the operation is simple and convenient, and it is easy to use. And there are also shortcomings: these operators are sensitive to noise which will greatly reduce the accuracy of image processing and affect the results. The noise sensitive degree of Canny operator is lowest, and as the computing complexity 
of Canny operator is higher when compared to the previous three operators, so the processing time of edge extraction is usually several times of the first three basic operators.[4]

According to the situation that cannot be ignored, in this paper, a method of image edge extraction based on line fitting is adopted to obtain the edge of steel cord pieces in factory production line.

\section{Edge detection based on line fitting}

\section{Threshold selection}

First, the original gray image is smoothed and some of the noise points is removed.Through the analysis of steel cord cutting edge scene captured by CCD camera and shown in figure 1, the conclusions can be drawn that the edge segmentation background and cutting piece target points are in the majority, while the boundary points whose gray value is approximately the threshold $g$ or equal to threshold $g$ are in the minority.If spurious interference and noise is considered, this condition is to use the gray value which has the least points as the threshold.This is common Gray Histogram Valley Method whose substance is that the background points and the target points account the majority, but boundary points close to the threshold( in histogram valleys) account the minority.[5,6,7]

Points whose gray value closes to the threshold point are rare, while there are many other points, which is to say that points where the gray value is $\mathrm{g}$ account small proportion $p_{g}$ in an image. Therefore the value of $1 / p_{g}$ is large and the gray-weighted average using $1 / p_{g}$ as weight coefficient can be as a threshold.

$$
g=\frac{\sum_{p_{i} \neq 0} i / p_{i}}{\sum_{p_{i} \neq 0} 1 / p_{i}}
$$

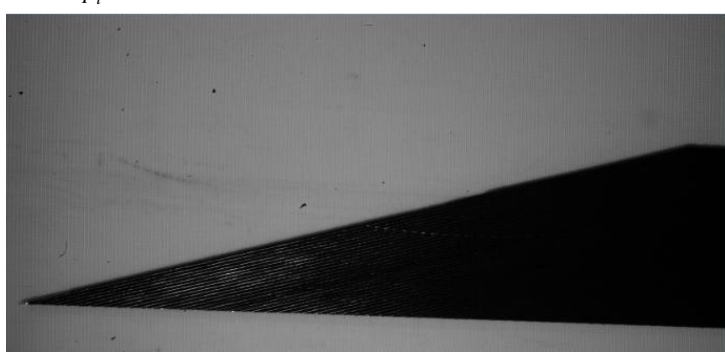

Figure 1 Product image

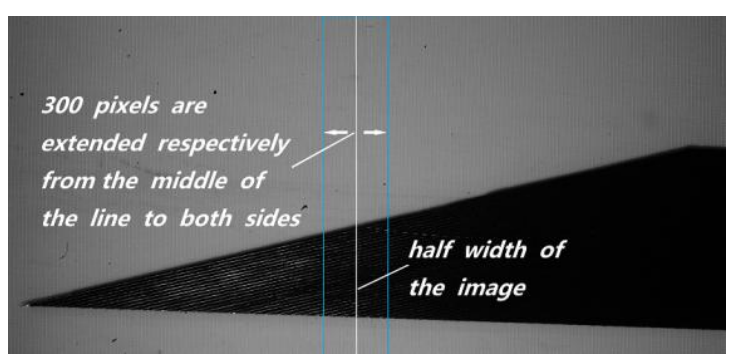

Figure 2 Selected area for analysis

Because horizontal pixel of the image is 6576 , and vertical pixel is 4384 , the entire image pixel is very much. If each pixel is taken, the methods of extracting pixel will increase the processing time. So based on the Gray Histogram Trough Method,vertical lines are done from top to bottom in position of $1 / 2,1 / 4,3 / 4$ of the image and then the lines are used as middle line to select analysis area which extends 300 pixels respectively on both sides. Those pixels are applied to calculate the threshold. Figure 2 shows the analysis area.

\section{Line fitting}

The gray value of points near the edge of image will has great changes. Point gradient whose gray value is close to the threshold is calculated. The larger the gradient of the point in the threshold is, the point is closer to the exact edge point. The calculation method that line fitting is combined with gradient calculation based on Sobel operator is more accurate to analyze the points for linear fitting. Gradient magnitude is calculated by $3 \times 3$ area shown in table 1 .

$$
M=\sqrt{S_{x}^{2}+S_{y}^{2}}
$$

Wherein the partial derivative $S x$ and Sy can be calculated by convolution mask.

$$
\begin{aligned}
& S_{x}=\left(Z_{1}+2 Z_{2}+Z_{3}\right)-\left(Z_{7}+2 Z_{8}+Z_{9}\right) \\
& S_{y}=\left(Z_{1}+2 Z_{4}+Z_{7}\right)-\left(Z_{3}+2 Z_{6}+Z_{9}\right)
\end{aligned}
$$


Table 1 Calculation templates

\begin{tabular}{|c|c|c|c|c|c|c|c|c|}
\hline \multicolumn{3}{|c|}{$\begin{array}{l}\text { Sobel operator vertical } \\
\text { template }\end{array}$} & \multicolumn{3}{|c|}{$\begin{array}{l}\text { Sobel operator horizontal } \\
\text { template }\end{array}$} & \multicolumn{3}{|c|}{ Image Template area } \\
\hline 1 & 2 & 1 & 1 & 0 & -1 & $\mathrm{Z}_{1}$ & $\mathrm{Z}_{2}$ & $\mathrm{Z}_{3}$ \\
\hline 0 & 0 & 0 & 2 & 0 & -2 & $\mathrm{Z}_{4}$ & $(\mathrm{x}, \mathrm{y})$ & $\mathrm{Z}_{6}$ \\
\hline-1 & -2 & -1 & 1 & 0 & -1 & $\mathrm{Z}_{7}$ & $\mathrm{Z}_{8}$ & $Z_{9}$ \\
\hline
\end{tabular}

As can be seen from Figure 1, goal edges are basically made up of several straight lines if flash is ignored, which is a polygon including three straight lines and picture edges. The image coordinate system is set where the origin is located in the image lower left corner, $x$ axis direction is horizontal to the right, and y direction is vertically upward. First collecting the points in the upper boundary: $x P o s$ which is the half width of the image is employed as abscissa and the first background point whose gray value less than the threshold value of $g$ and which is encountered from the top to the bottom in the vertical line is the demarcation point. Then this point is as the center. The gradient $M$ in this point is calculated by sobel operator. Then gradients $M i$ of ten points from the surrounding are calculated. If $M i>M$,the point with large gradient is stored into the array.Next $x P o s$ is as the center. Eligible points are accessed by traversal search from left to right. Similarly, the first vertical decomposition point is sought from the bottom to the top to collect the points in lower boundary.

Make fitting line: $f(x)=k x+b$. Apply least square method to calculate the parameters $k$ and $b . \mathrm{N}$ represents the number of discrete eligible points and can make the squared error sum $\mathrm{M}$ to be the smallest.[8]

$$
M=\sum_{i=1}^{N}\left[y_{i}-\left(k x_{i}+b\right)\right]^{2}
$$

Concrete operation steps are as follows:

1) Solve the partial derivative of $k$ and $b$ respectively. And make the value of partial derivative function to be zero:

$$
\left\{\begin{array}{l}
\frac{\partial M}{\partial k}=-2 \sum_{i=1}^{N}\left[y_{i}-\left(k x_{i}+b\right)\right] x_{i}=0 \\
\frac{\partial M}{\partial b}=-2 \sum_{i=1}^{N}\left[y_{i}-\left(k x_{i}+b\right)\right]=0
\end{array}\right.
$$

2) Rearrange and combine the bracket parameter in formula (7).Separate the unknown number k and b. We can get:

$$
\left\{\begin{array}{c}
k \sum_{i=1}^{N} x_{i}^{2}+b \sum_{i=1}^{N} x_{i}=\sum_{i=1}^{N} y_{i} x_{i}, \\
k \sum_{i=1}^{N} x_{i}+N b=\sum_{i=1}^{N} y_{i} ;
\end{array}\right.
$$

3) Solution of equations are obtained:

$$
\left\{\begin{array}{c}
k=\frac{\sum_{i=1}^{N} x_{i} \sum_{i=1}^{N} y_{i}-N \sum_{i=1}^{N} x_{i} y_{i}}{\sum_{i=1}^{N} x_{i} \sum_{i=1}^{N} x_{i}-N \sum_{i=1}^{N} x_{i} x_{i}} \\
b=\frac{\sum_{i=1}^{N} y_{i}-k \sum_{i=1}^{N} x_{i}}{N}
\end{array}\right.
$$

Edge fitting straight line equation is: $f(x)=k x+b$.

The linear relationship between the cutting block obtained edge points is roughly calculated. Through traversing each point within the two arrays, straight line fitting is accomplished and fitting line is obtained.

In order to improve the detection accuracy of straight line, influence of the noise points near the 
line need be reduced. As shown in figure 3,the points far from the line have great impact on the fitting process.

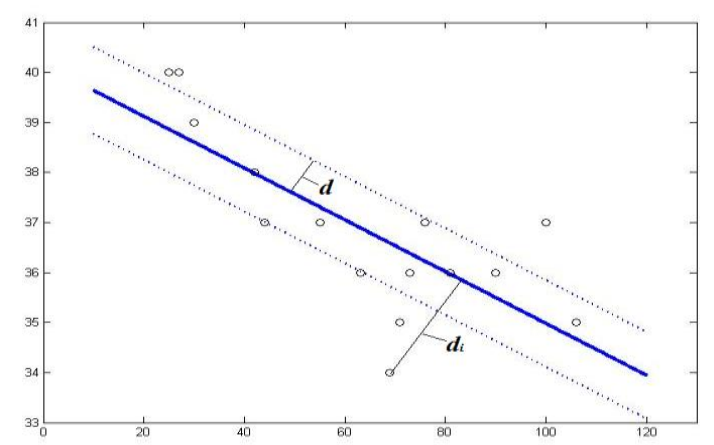

Figure 3 Discrete points near the fitting line

Calculate the distance from array points $d_{i}$ to the fitting line and calculate average distance $d$. The results are shown in formula (10) and (11).

$$
\begin{aligned}
& d_{i}=\left|\frac{k x_{i}-y_{i}+b}{\sqrt{k^{2}+1}}\right| \\
& d=\frac{1}{N} \sum_{i=1}^{N} d_{i}
\end{aligned}
$$

Set a value $m$. If a point $d_{i}$ satisfies the inequality $d_{i}>m$, it will be deleted. Filter out the discrete points far from the line and Fit a straight line again.

Image edge detection can be realized by the fitting line and detection data also can be obtained, including the cutting edge length(the image hypotenuse) and angle of steel wire cord cutting block. The steps are probably as follows:

input: Length $=$ The number of image horizontal pixels; height $=$ The number of image vertical pixels; $\mathrm{g}=$ Threshold value; $\mathrm{a}=$ Gray value;

Output: hypotenuse = Hypotenuse length: hypotenuse; angle = The minimum angle;

Look for all fitting lines in upper and lower boundary;

Fit a straight line;

Extract gray value of pixels through shard sampling and calculate threshold g;

When $\mathrm{x}=1 / 2$ length, for $(\mathrm{y}=0 ;, \mathrm{y}<$ height; $\mathrm{y}++)$

If gray value a $<$ threshold $g$

For find from the specified point and from left to right

For find within the scope of ten points from top to bottom

If Gradient value $\mathrm{M}_{1}<$ Gradient value $\mathrm{M}$

Then set as the reference point on upper boundary;

End

\section{End}

Points in the array is done linear fitting on the condition that every five points are divided into a group; line;

Calculate the difference slope of the two straight line slope, if slope $<1$, it is a straight

Look for unqualified points in turn from nearby upper and lower boundary;

If the distance between the first points and fitting line $d_{i}>$ the average $d$

distance from all points to fitting line

Then delete this point and form new array;

\section{End}

Do line fitting again by new array;

Calculate the sine value between two straight lines $\left(k_{1}-k_{2}\right) /\left(1+k_{1} * k_{2}\right)$ and judge whether two lines intersect; 
Calculate the intersection of two straight lines.Calculate the distance between two points.Calculate the hypotenuse length hypotenuse and angle angle.

\section{Experimental results and analysis}

In this paper, the experimental program uses $\mathrm{C}++$, and debug them successfully in the $\mathrm{C}++6.0$ Visual environment. The steel cord pieces detection platform built in factory is as shown in Figure 4, which can collect images on the spot. Using industrial CCD camera (Figure 5) obtain real-time image in the external trigger, and the image will be sent to the computer for image processing [9].

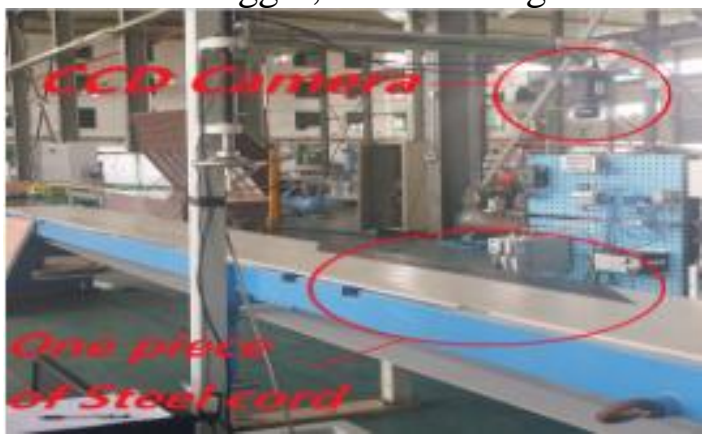

Figure 4 The steel cord pieces detection platform

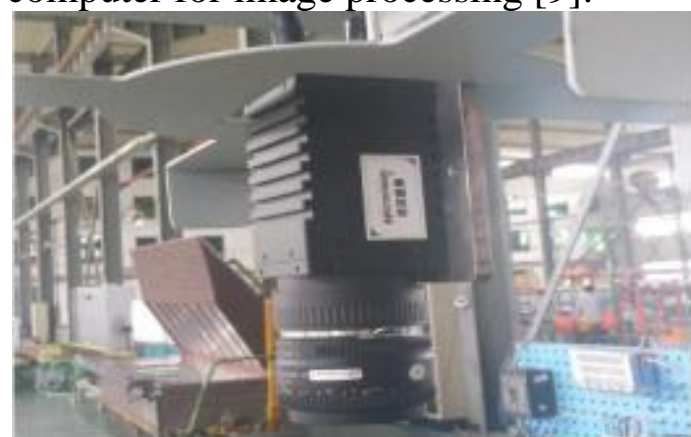

Figure 5 The industrial CCD camera

In the experiment, we select the measured image of steel cord piece in Figure 1, take the method of line fitting to do the edge processing for the image. We then extract the edge of the steel cord piece, and compute the required hypotenuse and angle by the extracted edge which is shown in Figure 7:

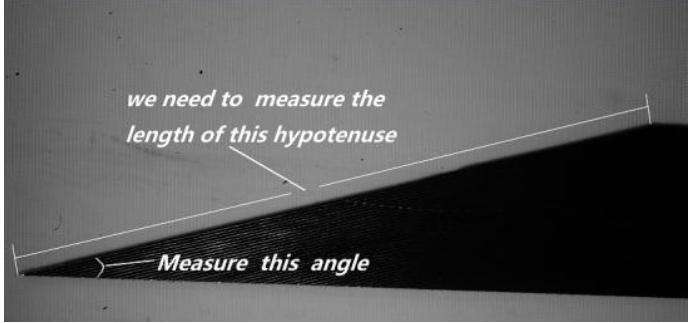

Figure 6 the prediction image

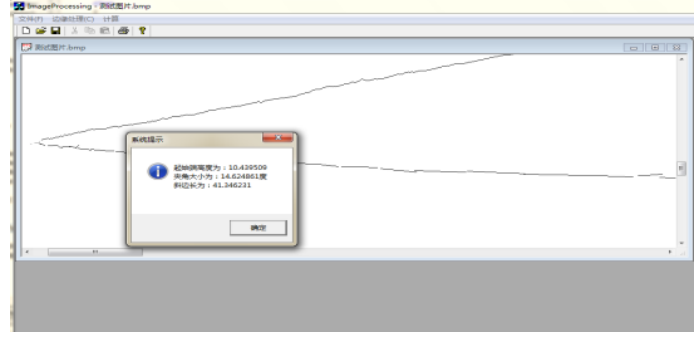

Figure 7 The show of edge after processing

The boundary points extracted by the straight line fitting method is composed of single pixel, as shown in Figure 8(a); compared with the results of the Sobel operator edge detection, as shown in Figure 8(b), we can see that using Sobel operator to deal with the edge, under the circumstances that the gray level of the image is not even, and there're more interference noise point, processing results is not clear and can only roughly recognize the edge, produce large calculation errors. In this paper, the algorithm can accurately extract edge straight line and the results are more accurate. Therefore, the method has high reliability of edge recognition and can effectively restrain the noise.

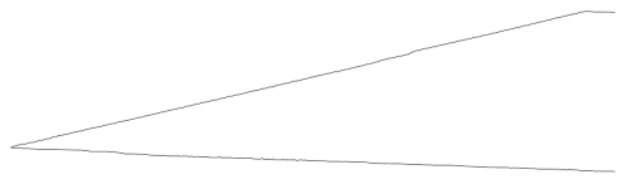

(a) The edge extraction result based on line fitting

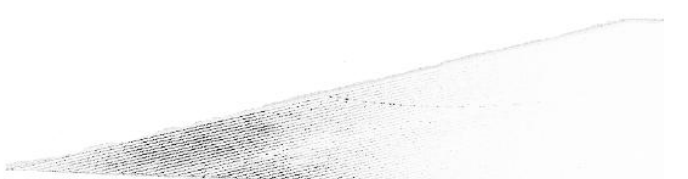

(b) The edge extraction result based on Sobel operator

Figure 8 the comparison of edge extraction result between the proposed algorithm and Sobel operator

Get images of the steel cord pieces several times on the conveyor belt, then obtain the results as shown in Table 2: 
Table 2 The test results

\begin{tabular}{|l|l|l|l|l|l|l|l|}
\hline & $\begin{array}{l}\text { Standard } \\
\text { size }\end{array}$ & $\begin{array}{l}\text { The } \\
\text { First } \\
\text { test }\end{array}$ & $\begin{array}{l}\text { The } \\
\text { Second } \\
\text { test }\end{array}$ & $\begin{array}{l}\text { The } \\
\text { Third } \\
\text { test }\end{array}$ & $\begin{array}{l}\text { The } \\
\text { Fourth } \\
\text { test }\end{array}$ & $\begin{array}{l}\text { The } \\
\text { Fifth } \\
\text { test }\end{array}$ & $\begin{array}{l}\text { The } \\
\text { maximum } \\
\text { relative error }\end{array}$ \\
\hline $\begin{array}{l}\text { The height of } \\
\text { steel cord } \\
\text { piece }(\mathrm{cm})\end{array}$ & 10.6 & 10.43 & 10.59 & 10.38 & 10.52 & 10.50 & 0.02 \\
\hline \begin{tabular}{l} 
The angle ( $)$ \\
\hline
\end{tabular} & 15 & 14.62 & 14.886 & 14.698 & 14.641 & 14.93 & 0.025 \\
\hline $\begin{array}{l}\text { the hypotenuse } \\
\text { length (cm) }\end{array}$ & 41 & 41.31 & 41.22 & 40.91 & 40.83 & 40.75 & 0.0075 \\
\hline
\end{tabular}

From Table 1 we can see, the accuracy of the hypotenuse length is basically controlled under $0.01 \mathrm{~cm}$ and angle precision is controlled below $0.03^{\circ}$. These reach the requirements that the detection length accuracy of steel cord pieces should be under $0.01 \mathrm{~cm}$.

\section{Conclusions}

Through the analysis and the experimental results we can see that, the line fitting is applied to the image edge detection which can effectively improve the accuracy and speed of the edge detection of steel cord pieces. The method combines the following characteristic of the detected image: the background points and the target points are in the majority and its shape is single. It uses Gray Histogram Valley Method, extracts data by taking a regional sampling method and computes the image threshold, all these can improve the speed of threshold selection. The method we use is finding the suspected edge points first and then taking these points as the center to use the Sobel operator by determining the gradient magnitude to give a relatively accurate edge points group, and fitting a straight line. Then calculate the distance from the point in the array to the fitting line, remove the discrete points which deviate the distance and use the line fitting again. The experimental results show that the method improves the speed and accuracy of the edge detection.

\section{Acknowledgements}

This research work was supported by National Natural Science Foundation Project(Fund Number:51365010).

\section{References}

[1] Josefina Perlo, Christoph Mülder, Ernesto Danieli. Digital processing of images of extruded rubber profiles for process control MRI[J]. Measurement., 82 (2016) 466-475 .

[2] Tao Li-mei. Steel Cord Calendering Cord Arrangement Detection System[J]. Tire Industry. 2014,03:184-187.

[3] FENG Wu, ZHANG Jun-lan, MIAO Qiu-jin。Evaluation of several typical edge detection operator[J]. Electronic Design Engineering. 2011,04:131-133.

[4] ZHANG Tai-fa,Gao Chao-yang. Analysis and Study of Edge Detection Approach in Digital Images[J]. Computer Technology And Development , 2010,10:141-145.

[5] Wu Xue-ming, LI Can-ping. Research on Performance of Edge Dectection Operators in Image Processing under Different resolutions[J].Computer Measurement \&Control ,2006,02:166-169.

[6] WANG Zhi-rui,YAN Cai-liang. Method of the Image Feature Exrraction[J]. Journal of Jishou University(Natural Sciences Edition), 2011,05:43-47. 
[7] FU Zhong liang. The Making of Method for Image Threshold Selection[J]. JOURNAL OF IMAGE AND GRAPHICS, 2000,06:21-24.

[8] NI Jin-hui, XIAO Jun,WEN Li-we. Real-time Visual Inspection Method to Detect Prepreg Edge Straight Based on Line Fitting[J].Computer Science ,2015,S1:16-19+23.

[9] LIU Fen-fei, ZHAO Hui, TAO Wei.Improved edge detection method for the linear CCD image on linear fitting[J]. Opto-electronic Engineering,2005,03:40-43. 